\title{
Determinantes da Distribuição da (Des)igualdade de Gênero entre os Estados Brasileiros
}

\author{
Adriana Bruscato Bortoluzzo \\ Professora Insper \\ Endereço: Rua Quatá, 300 - São Paulo/SP \\ CEP: 04546042 -E-mail: adrianab@insper.edu.br \\ Ieda Rodrigues Matavelli \\ Professora Insper \\ Endereço: Rua Quatá, 300 - São Paulo/SP \\ CEP: 04546042 - E-mail: iedarm@al.insper.edu.br \\ Regina Madalozzo \\ Professora Insper \\ Endereço: Rua Quatá, 300 - São Paulo/SP \\ CEP: 04546042 - E-mail: reginam@insper.edu.br
}

Recebido em 12 de dezembro de 2014. Aceito em 06 de setembro de 2015.

\section{Resumo}

A igualdade de gênero é fundamental para um desenvolvimento econômico sustentável. O Brasil, apesar de ser uma das maiores economias do mundo, ainda precisa de um grande avanço no sentido de conquistar uma sociedade mais igualitária entre homens e mulheres quando comparado a países do mesmo porte econômico. O presente estudo faz uma análise da evolução da igualdade de gênero no Brasil entre 1997 e 2012, e quais os impactos da globalização, do desenvolvimento econômico e de medidas de bem-estar sobre a distribuição da igualdade de gênero entre os estados brasileiros. Para isso, foi utilizada a metodologia econométrica de dados em painel. Os resultados mostram que a igualdade de gênero aumentou no período e que, além disso, a globalização, mensurada com base no investimento direto estrangeiro e no acesso da população a aparelhos de televisão, elevou a igualdade entre os sexos no Brasil.

\section{Palavras-Chave}

Igualdade de gênero. Dados em painel. Estados brasileiros.

\begin{abstract}
Gender equality is crucial for sustainable economic development. Brazil, despite being one of the largest economies in the world, is far from being considered an egalitarian society between men and women when compared to the same economic size countries. This study analyzes the evolution of gender equality in Brazil between 1997 and 2012 and the impacts of globalization, economic development and welfare measures on the distribution of gender equality among States. In order to reach this goal, we used the econometric methodology of panel data. The results show that gender equality has increased over the period and that globalization, measured based on foreign direct investment and on the population's access to television sets, raised the gender equality in Brazil.
\end{abstract}

\section{Keywords}

Gender equality. Panel data. Brazilian states.

\section{JEL Classification}

J16. J33. J71. 


\section{Introdução}

De acordo com o Fundo de População das Nações Unidas (UNFPA, United Nations Population Fund), o avanço dos direitos das mulheres contribui positivamente para a saúde e produtividade das famílias e comunidades, além de beneficiar as gerações futuras. Investir em políticas públicas voltadas para o desenvolvimento da mulher é racional do ponto de vista econômico e vital para elevar o bem-estar social. Segundo dados do Banco Mundial, recursos administrados por mulheres aumentam os gastos em áreas que beneficiam as crianças. Além disso, cada ano adicional na escolaridade das garotas pode aumentar a sua renda futura entre $10 \%$ e $20 \%$. Apesar de um significante avanço nos últimos anos, muitas mulheres ainda não possuem igualdade tanto com relação aos direitos de liberdade como nas oportunidades com relação aos homens, enfrentando grande discriminação na sociedade como um todo (Branisa et al., 2014), no mercado de trabalho (Weichselbaumer e Winter-Ebmer, 2005) e na educação (Senadza, 2012), o que retarda o desenvolvimento econômico (Knowles, Lorgelly e Owen, 2002).

No contexto brasileiro, houve um avanço recente no sentido de promover a igualdade entre os sexos. O país passou da $85^{\mathrm{a}}$ posição em 2010 para a $71^{\text {a }}$ em 2014, num total de 142 países no ranking por nível de equidade de gênero desenvolvido pelo Fórum Econômico Mundial. ${ }^{1}$ Ainda assim, por ser a sétima maior economia do mundo (FMI, 2013), o Brasil tem a responsabilidade e a oportunidade de se destacar perante países do mesmo porte ao investir na formulação de políticas públicas voltadas para o desenvolvimento da mulher e, com isso, atingir um desenvolvimento sustentável no longo prazo.

A desigualdade de gênero pode ser mensurada de diferentes maneiras. Sen (2001) a define como uma ligação de diferentes dimensões e não como um fenômeno homogêneo. Essa disparidade pode ser, por exemplo, desencadeada por vários fatores, como desigualdade na participação política, no acesso à educação e à saúde, de salários ou até mesmo na taxa de natalidade. Baseados na metodologia utilizada pelo Fórum Econômico Mundial para comparar os diversos países na

1 O índice calculado pelo Fórum Econômico Mundial contempla quatro diferentes categorias que representam as diferenças entre os gêneros: participação econômica, educação, participação política e saúde. 
questão de equidade de gênero, Ramos e Souza $(2012)^{2}$ construíram um índice nacional - para os estados brasileiros.

Os objetivos deste trabalho consistem em analisar a evolução da (des)igualdade de gênero no Brasil entre 1997 e 2012, e em estudar a distribuição desta entre os estados brasileiros, dada a dimensão continental do país. Assim, será utilizada a abordagem econométrica de dados em painel para verificar como a (des)igualdade de gênero respondeu ao longo do tempo à globalização, ao desenvolvimento econômico e a medidas de bem-estar. Os resultados mais relevantes deste estudo mostram que houve aumento significativo na igualdade de gênero no Brasil no período estudado, e que este aumento deve-se principalmente ao crescimento do Investimento Direto Estrangeiro (IED), do percentual de domicílios com acesso à televisão e da diminuição da pobreza, enquanto o gasto do governo contribui negativamente para a melhora da igualdade de gênero.

Este artigo está estruturado da seguinte maneira: a seção 2 contém uma revisão da literatura de desigualdade de gênero, concentrandose nos fatores determinantes da desigualdade para diferentes países. A seção 3 descreve as bases de dados e a metodologia econométrica adotadas. Os resultados são analisados na seção 4 e, por fim, a seção 5 conclui o artigo apresenta recomendações para estudos futuros.

\section{Revisão da literatura}

A temática que gira em torno das questões relacionadas aos diferenciais de gênero tem sido explorada cada vez mais na literatura acadêmica. Tradicionalmente, teorias relacionadas ao mercado de trabalho (Addison and Demet Oztuk, 2012) e diferenças salariais (Miki and Fany, 2011) eram as mais frequentes. Entretanto, com o aumento da disponibilidade de dados e métodos alternativos de investigação, nos últimos anos houve aumento do interesse acadêmico em outras questões de gênero como a distribuição de poder familiar (Ham and Song, 2014), a presença de mulheres em cargos de liderança nas em-

2 Em Ramos e Souza (2012), o índice foi denominado "Índice Nacional da Desigualdade de Gênero". Entretanto, o índice assume valores entre 0 (zero) e 1 (um), onde 0 (zero) indica desigualdade total entre os gêneros e 1 (um) significa a igualdade. Dessa forma, o índice mede a igualdade e não a desigualdade de gênero e, por esse motivo, doravante será denominado "Índice da Igualdade de Gênero" (IIG) no presente estudo. 
presas e na política (Wang and Kelan, 2013) e até mesmo na questão da (des)igualdade entre os gêneros (Branisa et al., 2014).

Como a questão de gênero contamina-se fortemente com questões culturais específicas, muitos estudos concentram-se em análises de regressões cross-country para explicar os causadores da (des)igualdade de gênero. Dessa forma, uma alternativa de linha de pesquisa é o foco acerca do papel que o desenvolvimento econômico exerce sobre a igualdade de gênero. Um dos estudos que optam por essa vertente é Dollar e Gatti (1999). Nesse estudo, os pesquisadores concluem, por meio de uma análise com dados em painel com efeitos fixos e utilizando uma amostra de 100 países de 1960 a 1990, que aumentos na renda per capita de um país levam a uma redução da desigualdade de gênero, argumentando que existem falhas de mercado que dificultam o investimento em garotas em países em desenvolvimento e que isso é geralmente superado na medida em que estes se desenvolvem.

Na mesma linha, Gumbel (2004) utiliza uma amostra de países desenvolvidos e industrializados na tentativa de diminuir os efeitos da influência regional, religiosa e do regime político para concluir, na mesma linha que Dollar e Gatti(1999), que uma maior renda per capita está associada a uma menor desigualdade de gênero. Além disso, também mostra que a desigualdade no acesso à educação relaciona-se forte e negativamente com altos níveis de Produto Nacional Bruto (PNB) per capita.

Por outro lado, Seguino (2006) conclui que crescimento econômico, num cenário recente de globalização, não levou à maior equidade de gênero em países da América Latina e da região do Caribe com dados de 1970 a 2000, questionando, dessa forma, a relação causal antes estabelecida entre crescimento econômico e (des)igualdade de gênero. A autora foca em indicadores relativos de desigualdade, como a taxa de analfabetismo de homem com relação à mulher, a taxa de fertilidade relativa e a proporção feminina na força de trabalho. Essas variáveis foram consolidadas num índice que mensura o nível de equidade no bem-estar dos diferentes países da amostra e que é a variável analisada no seu modelo, analogamente ao que será feito com o Índice de Igualdade de Gênero (IGG) neste trabalho. Assim, apesar de mostrar que crescimento econômico não foi responsável por uma melhora na desigualdade de gênero, a autora argumenta que os gastos governamentais, como um mecanismo de redistribuição de 
renda por meio de dispêndios com políticas sociais, influenciaram positivamente o bem-estar das mulheres.

Com um foco maior nos impactos da globalização na desigualdade de gênero, alguns estudos concluem também que os efeitos da liberalização comercial podem variar de acordo com o nível de desenvolvimento dos países, uma vez que países emergentes e desenvolvidos possuem diferentes estruturas sociais, econômicas e institucionais. Baliamoune-Lutz (2006) é um desses estudos. Utilizando uma amostra de países africanos, mostra que a abertura comercial levou a um aumento da desigualdade de gênero. Isso porque uma maior abertura leva a um aumento da oferta de trabalhadores não qualificados, elevando a desigualdade. Já Oostendorp (2004) examina a desigualdade salarial entre homens e mulheres desde o início do processo de globalização em países em desenvolvimento. A autora encontra que os efeitos da maior abertura comercial e de maiores fluxos de investimento estrangeiro direto sobre a desigualdade de gênero são incertos.

Juhn, Ujhelyi e Villegas-Sanchez (2013) analisam quais os impactos do NAFTA, acordo comercial entre México e Estados Unidos, na desigualdade salarial entre homens e mulheres no México. Concluem que uma redução das tarifas devido à implementação do NAFTA induziu as firmas mais produtivas a modernizarem suas tecnologias e a entrarem no mercado de exportação. Com isso, o salário relativo e a empregabilidade cresceram entre as mulheres que compunham a força de trabalho operária, mas não afetou significativamente as que trabalhavam em cargos administrativos.

Arora (2012) verifica quais os impactos da globalização e do desenvolvimento econômico na desigualdade de gênero para os diferentes estados da Índia. O autor mostra que, ao mesmo tempo em que estados com elevada renda per capita possuem baixa desigualdade de gênero, existem também alguns estados em que uma alta renda per capita coincide com alta desigualdade, de modo que renda elevada não parece ser determinante para equidade de gênero. Com relação à globalização, o estudo mostra que a maioria dos estados com alto grau de abertura possui desigualdade alta. Estas conclusões baseiamse em análises de correlações entre as variáveis, uma vez que o autor não propõe uma abordagem econométrica para verificar a relação entre as variáveis. 
Estudos acerca de dados brasileiros ou que envolvam o Brasil em suas amostras são mais raros. Conforme já mencionado, existe uma concentração de estudos com relação aos diferenciais de salário por gênero, onde se encaixam Giuberti e Menezes-Filho (2005) e Madalozzo (2010). Madalozzo (2010) utiliza a metodologia de decomposição de Oaxaca para estudar o diferencial de salários de homens e mulheres entre os anos 1978 e 2007. Os resultados mostram que, no geral, a remuneração das mulheres é mais baixa que a dos homens, quando controlada por características individuais, mas que essa desigualdade reduziu de 33\% em 1987 para 15,4\% em 2007.

Na mesma linha, Giuberti e Menezes-Filho (2005) também estudam a discriminação salarial entre homens e mulheres no Brasil e nos Estados Unidos entre 1981 e 1986. Concluem que, em ambos os países, houve uma redução da desigualdade salarial entre gêneros no período, mas que ainda há parte dessa desigualdade que não é explicada por características individuais, o que viria a caracterizar a discriminação sofrida pelas mulheres no mercado de trabalho.

Seguindo a ótica da desigualdade de gênero do ponto de vista de estrutura social, diversos estudos têm foco nas relações entre homens e mulheres em diferentes esferas no Brasil. Rosa (2009) foca na dicotomia raça e gênero para explicar diferenças socioeconômicas das famílias. Ramos (2009) volta ao foco de discriminação com relação às mulheres, mas trazendo à tona as questões da distribuição do uso de tempo. Como a definição dos padrões de tempo dedicado ao trabalho remunerado e não remunerado seguem uma dinâmica muito próxima às relações de poder com relação ao gênero, esse estudo enfatiza a necessidade de políticas públicas que oportunizem a maior valorização das questões e impasses de conciliação de tempo principalmente com relação às escolhas das mulheres.

O estudo com maior relação com a pesquisa atual é Ramos e Souza (2012). Os autores constroem um Índice Nacional de Desigualdade de Gênero (INDG) para comparar a desigualdade entre os diferentes estados brasileiros baseado no Global Gender Gap Index (GGI), desenvolvido pelo Fórum Econômico Mundial. O índice é composto por quatro categorias diferentes - participação econômica, educação, poder político e saúde/sobrevivência. Replicado para o Brasil, o INDG foi calculado com base nos dados da PNAD do ano de 2009. Ramos e Souza (2012) concluem que os estados do Norte-Nordeste 
obtiveram melhores pontuações no índice do que os estados do SulSudeste, indicando que a renda parece não ser um determinante direto de uma maior equidade de gênero. Assim, considerando o INDG como variável de interesse - e agora o renomeando para Índice de Igualdade de Gênero, IIG - o presente estudo propõe uma modelagem econométrica para verificar os determinantes da (des) igualdade de gênero no país.

\section{Metodologia}

Nesta seção estão descritas detalhadamente as variáveis utilizadas no trabalho e o modelo econométrico que avalia a relação entre a igualdade de gênero e seus fatores determinantes nos estados brasileiros no período de 1997 a 2012.

\subsection{Variável Dependente}

$\mathrm{Na}$ literatura, a maioria dos estudos empíricos analisa a desigualdade de gênero em um único aspecto, seja ela no mercado de trabalho, na participação política ou no acesso à educação. Entretanto, a desigualdade entre homens e mulheres acontece em diferentes dimensões, surgindo a necessidade de um indicador que englobe todas elas para mensurá-la de maneira mais exata.

Com foco na criação de um indicador deste tipo, em 1995, o Programa das Nações Unidas para o Desenvolvimento (PNUD) elaborou o Gender-related Development Index (GDI) e o Gender Empowerment Measure (GEM). Por serem pioneiros, esses indicadores tiveram elevada importância (tanto no meio acadêmico quanto não acadêmico) por conscientizar sobre questões relacionadas à desigualdade de gênero. Entretanto, apesar de relevantes, foram muito criticados por suas limitações conceituais e metodológicas, uma vez que o GDI é considerado um índice de desenvolvimento, e não de desigualdade, enquanto o GEM restringe sua análise à determinada classe social. Em decorrência disso, o PNUD introduziu um novo indicador em 2010, o Gender Inequality Index (GII), que contempla três diferentes dimensões: empowerment, atividade econômica e saúde reprodutiva. Porém, a complexidade excessiva, e até mesmo 
desnecessária, de sua fórmula e a incorporação de medidas absolutas (específicas à mulher) e relativas (mulher versus homens), levam a problemas conceituais e metodológicos, além de dificultarem a interpretação (Permanyer, 2013).

Existem também indicadores que focam em aspectos institucionais da desigualdade, baseado em tradições e normas sociais, como o Social Institutions and Gender Index (SIGI) da Organização para a Cooperação e Desenvolvimento Econômico (OECD), de 2009, e o Women's Economic Opportunities Index (WEOI), desenvolvido pela Economist Intelligence Unit em 2010. Contudo, apesar de relevante, a questão institucional é de difícil mensuração para os diferentes estados brasileiros e, por esse motivo, não será considerada na variável de interesse deste estudo.

Atualmente, o índice mais divulgado e amplamente utilizado para a comparação da desigualdade entre os gêneros para diferentes países é o Global Gender Gap Index (GGI), desenvolvido anualmente desde 2006 pelo Fórum Econômico Mundial para cerca de 140 países. Este índice utiliza medidas qualitativas - como políticas nacionais - bem como estatísticas internacionais e dados de pesquisas provenientes da Organização Internacional do Trabalho (OIT), UNESCO, entre outras instituições. Ao ranquear os países com relação à desigualdade entre homens e mulheres, o índice induz à criação de políticas e métricas internas que signifiquem medidas efetivas para redução dessa desigualdade. Este indicador contempla a desigualdade de gênero em quatro diferentes aspectos, sendo eles economia, política, educação e saúde, além de combinar dados quantitativos e qualitativos, obtidos pela Executive Opinion Survey, também do Fórum Econômico Mundial. Importante ressaltar que se trata de um índice de desigualdade, não especificamente de desenvolvimento. Desse modo, o índice mensura a situação relativa entre mulheres e homens no acesso aos recursos e às oportunidades num determinado país, e não o nível de recursos e oportunidades existentes.

Baseado no GGI descrito acima e em Ramos e Souza (2012), o presente estudo constrói, para os diferentes estados brasileiros, o Indice de Igualdade de Gênero (IIG), que será a variável de interesse do modelo econométrico. A principal base de dados utilizada para a construção do índice foi a Pesquisa Nacional de Amostra por Domicílio (PNAD) para os anos 1997, 2002, 2007 e 2012, reali- 
zada pelo Instituto Brasileiro de Geografia e Estatística (IBGE). Somente as variáveis referentes à dimensão Poder Político e Saúde/ Sobrevivência foram calculadas, respectivamente, com informações disponibilizadas no site do Tribunal Superior Eleitoral (TSE) e do IBGE. A Tabela 1 resume todas as variáveis (subíndices) utilizadas para a construção do IIG e suas respectivas dimensões.

Para o cálculo das dimensões Participação Econômica e Oportunidades e Educação, os microdados das PNADs foram desagregados por unidade da federação e sexo, ponderados pelo respectivo peso amostral. ${ }^{3}$ A categoria Participação Econômica e Oportunidades contempla três diferentes conceitos: disparidade na taxa de participação, na remuneração e no cargo profissional que cada um dos indivíduos ocupa. O primeiro consiste na razão entre a taxa de participação feminina e a masculina na força de trabalho, obtida pela divisão entre a População Economicamente Ativa (PEA) e a População em Idade Ativa (PIA). O segundo é representado pela razão entre o salário médio por hora de homens e mulheres no mercado de trabalho como um todo e também entre aqueles que ocupam a mesma posição (dirigentes e profissionais técnicos). O terceiro conceito engloba a razão entre a quantidade de mulheres e homens técnicos e também entre os que ocupam altos cargos. Uma falha dos indicadores dessa categoria é não capturar dados da economia informal, um setor significante na economia brasileira e que é sobre representado pelas mulheres (Folbre, 2006).

3 Para o cálculo da Participação Econômica, foram considerados apenas indivíduos com 16 anos ou mais, a fim de limitar o efeito de trabalho infantil que não auxilia na redução de desigualdades sociais nem de gênero. 
Tabela 1 - Descrição da composição do Índice Nacional de Desigualdade de Gênero (IIG).

\begin{tabular}{|c|c|c|}
\hline Índices & Subíndices & Fonte \\
\hline \multirow{5}{*}{$\begin{array}{c}\text { Participação } \\
\text { Econômica e } \\
\text { Oportunidades }\end{array}$} & $\begin{array}{l}\text { Razão entre a participacão feminina e a masculina no mercado } \\
\text { de trabalho }\end{array}$ & PNAD - IBGE \\
\hline & Razão entre o salário médio estimado de mulheres e de homens & PNAD - IBGE \\
\hline & $\begin{array}{l}\text { Razão entre a quantidade de mulheres e de homens que ocupam } \\
\text { altos cargos }\end{array}$ & PNAD - IBGE \\
\hline & $\begin{array}{l}\text { Razão entre a quantidade de profissionais técnicos mulheres e } \\
\text { homens }\end{array}$ & PNAD - IBGE \\
\hline & $\begin{array}{l}\text { Razão entre o salário médio estimado de mulheres e de homens } \\
\text { ocupando a mesma posição (dirigentes e profissionais técnicos) }\end{array}$ & PNAD - IBGE \\
\hline \multirow{4}{*}{ Educação } & Razão entre a quantidade de mulheres e de homens alfabetizados & PNAD - IBGE \\
\hline & $\begin{array}{l}\text { Razão entre a matrícula líquida de meninas e de meninos no en- } \\
\text { sino fundamental }\end{array}$ & PNAD - IBGE \\
\hline & $\begin{array}{l}\text { Razão entre a matrícula líquida de meninas e de meninos no en- } \\
\text { sino médio }\end{array}$ & PNAD - IBGE \\
\hline & $\begin{array}{l}\text { Razão entre a matrícula bruta de mulheres e de homens no ensino } \\
\text { superior }\end{array}$ & PNAD - IBGE \\
\hline \multirow{2}{*}{$\begin{array}{l}\text { Saúde/ } \\
\text { Sobrevivência }\end{array}$} & Razão entre o número de nascimentos de meninas e de meninos & IBGE \\
\hline & Razão entre a expectativa de vida feminina e a masculina & IBGE \\
\hline \multirow{2}{*}{ Poder Político } & $\begin{array}{l}\text { Razão entre a quantidade de mulheres e de homens parlamentares } \\
\text { (deputados federais e estaduais) }\end{array}$ & $\begin{array}{c}\text { Tribunal } \\
\text { Superior } \\
\text { Eleitoral (TSE) }\end{array}$ \\
\hline & $\begin{array}{l}\text { Razão entre a quantidade de mulheres e homens governadores } \\
\text { ou senadores }\end{array}$ & $\begin{array}{c}\text { Tribunal } \\
\text { Superior } \\
\text { Eleitoral (TSE) }\end{array}$ \\
\hline
\end{tabular}

Fonte: elaboração própria.

Na dimensão Educação, a desigualdade entre mulheres e homens é refletida pela razão entre a matrícula líquida de mulheres e homens nos ensinos fundamental, médio e superior. Também se considera a razão entre a quantidade de mulheres e homens alfabetizados como uma medida de longo prazo para verificar a eficácia do sistema educacional brasileiro em aumentar o capital humano de mulheres e homens de maneira similar.

Na categoria Saúde e Sobrevivência, existem dois subíndices. O primeiro é a taxa entre o número de nascimentos de meninas e meninos, com o intuito de capturar preferências por filho homem. O segundo é a razão entre a expectativa de vida da mulher pela do homem, a fim de considerar a diferença nos anos esperados de vida 
devido a fatores como violência, doenças, desnutrição, entre outros. Como no Global Gender Gap Index (GGI) desenvolvido pelo Fórum Econômico Mundial, o IIG estabelece marcos de igualdade de 0,94 e 1,06 para os subíndices nascimento e expectativa de vida respectivamente. Isto é, se a razão entre o número de nascimentos de meninas e de meninos for igual a 0,94 , esta será normalizada para 1 .

Na dimensão Poder Político, considera-se a razão entre a quantidade de mulheres e homens parlamentares (deputados federais e estaduais) e a razão entre a quantidade de mulheres e homens governadores ou senadores. Neste último subíndice, atribuímos o valor 1 caso fosse uma governadora (ou senadora) e 0 em caso contrário. Em determinados anos, porém, havia dois senadores por estado, então caso fosse um homem e uma mulher, a variável assumia 0,5. Por fim, foi feita a média aritmética entre a razão mulher/homem senador e mulher/homem governador para se chegar ao valor deste último subíndice. Neste índice foram feitas alterações na metodologia com relação ao índice desenvolvido por Ramos e Souza (2012). Ramos e Souza (2012) consideram como parte do índice de política a razão do número de mulheres que ocupam cargos eleitos no congresso nacional com relação ao número de homens. Considerando que o número de mulheres que se candidata a cargos no congresso nacional é bastante inferior ao número de candidatos homens e que isso pode impactar de forma significante a composição da dimensão política, fizemos uma adaptação nesse quesito. Em nosso índice, consideramos a razão do percentual de mulheres eleitas com relação ao número de candidatas sobre o percentual de homens eleitos com relação ao número de candidatos, o que revela a preferência do eleitor também com relação ao gênero. ${ }^{4} \mathrm{O}$ intuito da categoria Poder Político é verificar a evolução da participação da mulher na decisão política. Uma desvantagem desse indicador é a ausência de variáveis que capturam diferenças na participação política a nível municipal, como a proporção de prefeitos e vereadores mulheres para homens num determinado estado.

Para se chegar ao valor final de cada dimensão, foi feita a média aritmética de cada subíndice. Analogamente, para obter o Índice de Igualdade de Gênero (IIG) total por estado, também foi feita a média de cada dimensão. Importante ressaltar que, como se trata da

\footnotetext{
${ }^{4}$ Mesmo assim, para fins de testar a robustez de nossas estimativas, calculamos o índice exatamente da forma como Ramos e Souza (2012). Discutiremos os resultados na seção 4.
} 
razão mulher/homem, quanto mais próximo de 1 for o IIG, maior é a igualdade de gênero. Como todo indicador sintético, o IIG possui algumas limitações, incluindo aquelas já citadas anteriormente. $\mathrm{O}$ índice não captura, por exemplo, dimensões referentes à posse de ativos, violência devido a questões de gênero e ao uso do tempo - o fato de que muitas mulheres possuem menos tempo de lazer e maiores estresse e exaustão física por executarem tarefas domésticas e cuidarem das crianças. Essas variáveis não foram incluídas por indisponibilidade de dados em alguns dos anos inseridos em nossa amostra.

\subsection{Modelagem empírico-teórica}

O índice de igualdade de gênero (IIG) contempla quatro fatores distintos, conforme discutido na seção anterior. Com o objetivo de entender fatores que explicariam a melhoria do IIG dentro dos estados brasileiros, neste artigo recorre-se à literatura anterior focada em diferenças na equidade de gênero ocasionadas por alterações em medidas de desenvolvimento econômico (Lagerlöf, 2003; Dollar and Gatti, 1999; Gumbel, 2004), globalização (Arora, 2012; La Ferrara, Chong e Duryea, 2012; Oostendorp, 2004; BaliamouneLutz, 2006; Juhn, Ujhelyi e Villegas-Sanchez, 2013; Mukhopadyay and Chaudhuri, 2013) e bem-estar (Schneider, 2013; Bárcena-Martín e Moro-Egido, 2013; Seguino, 2006).

A modelagem teórica implica uma função de igualdade de gênero composta por fatores ligados a cada uma dessas categorias:

FIIG(C, CI, DSE, Pob, CE)

Onde:

FIIG é a função que compõe fatores explicativos para o IIG,

C representa a Cultura ou Abertura Cultural, proxy para globalização,

CI é o nível de comércio internacional, também ligado à variável de globalização,

DSE é o indicador de desigualdade econômica, 
Pob é a variável de percentual de pobreza e

CE representa o crescimento econômico, proxy para o indicador de desenvolvimento econômico do estado.

Segundo Arora (2012), ${ }^{5}$ o mecanismo de transmissão de maior igualdade de gênero via abertura cultural se faz a partir da permeabilidade de novas culturas frente ao status quo já estabelecido. Com um resultado na mesma linha, La Ferrara, Chong e Duryea (2012) mostram que, no Brasil, a disponibilidade de acesso a telenovelas teve um efeito nos índices de fertilidade: a identificação das mulheres - especialmente as com idade retratadas nas novelas - fez com que o comportamento retratado nas famílias das telenovelas - de maior independência das mulheres e menor taxa de fertilidade - fosse mimetizado na realidade das espectadoras. Dessa forma, a permeabilidade da cultura permitiu que o status quo dominante fosse alterado indiretamente por um fator extrínseco. Da mesma forma, espera-se que o grau de abertura cultural possa ter um forte impacto na demanda por maior igualdade de gênero nos estados. Para essa variável, utilizamos o percentual de domicílios que possuem televisão, calculado por meio dos microdados da PNAD, para captar o acesso à informação nos diferentes estados brasileiros e sua possível permeabilidade para mudanças culturais. Como outra medida que capture a dimensão cultural, também utilizamos uma variável relativa ao percentual de divórcios não consensuais solicitados por mulheres em cada estado. Essa variável tem potencial poder de explicação de menor vulnerabilidade ao poder patriarcal dentro da família e, com isso, impactar positivamente a igualdade entre os gêneros. ${ }^{6}$

Em linha com a abertura cultural, a permeabilidade da globalização via comércio internacional pode impactar positivamente a equidade entre os gêneros, conforme verificado em Mukhopadhyay e Chaudhuri (2013). Especificamente na questão de fluxo de capital e impacto nas relações de gênero, Mukhopadhyay e Chaudhuri

\footnotetext{
5 Para a exposição teórica da modelagem, a opção foi por seguir somente um dos autores para cada um dos fatores. Entretanto, a linha explicitada pelo autor escolhido está em consonância com os demais citados anteriormente.

6 Agradecemos a um(a) parecerista anônimo que nos incitou a procurar outras variáveis que melhor representassem a dimensão cultural nos diferentes estados. A variável relativa aos pedidos de divórcio foi a única disponível para todos os anos pesquisados e, desta forma, incluída em nossos modelos.
} 
(2013) se utilizam de um modelo baseado em diferenças salariais e de habilidade:

$$
\mathrm{W}_{\mathrm{I}}=\left(\mathrm{W}_{\mathrm{M}} \mathrm{H}-\mathrm{W}_{\mathrm{F}} \mathrm{h}\right)
$$

Onde:

$\mathrm{W}_{\mathrm{I}}$ representa a diferença salarial entre os gêneros,

$\mathrm{W}_{\mathrm{i}}$ é o salário de homens $(\mathrm{i}=\mathrm{M})$ ou mulheres $(\mathrm{i}=\mathrm{F})$,

H é a eficiência média dos homens e

h é a eficiência média das mulheres.

A partir dessa equação inicial, Mukhopadhyay e Chaudhuri (2013) concluem que a maior (des)igualdade entre os gêneros estaria atrelada ao tipo de capital que teria entrada no país. Fluxos de capital que privilegiam o setor ocupacional onde as mulheres teriam maior participação como mão de obra (e, através disso, elas possam ter ganho real de remuneração pela maior demanda por seu trabalho) gerariam uma diminuição na desigualdade salarial entre os gêneros. Em direção oposta, fluxo de capital estrangeiro que privilegie o mercado de trabalho com empregados essencialmente do sexo masculino teria efeito contrário.

Para verificar o efeito de comércio internacional na igualdade de gêneros em cada um dos estados brasileiros, utilizamos duas variáveis: o fluxo de investimento estrangeiro direto (IED) e o volume de comércio para cada estado. O IED foi coletado no Censo de Capitais Estrangeiros do Banco Central do Brasil para os anos 1995, 2000, 2005 e 2010 e deflacionado pelo índice de preços dos EUA, uma vez que a variável encontra-se em dólar. Bortoluzzo, Sakurai e Bortoluzzo (2013) mostraram que o IED se distribui de forma heterogênea nos estados brasileiros e que este fluxo pode representar a atratividade de investimento estrangeiro, ou seja, indica o nível de globalização de cada estado do país. Para evitar o problema de endogeneidade (simultaneidade) entre o investimento estrangeiro e a desigualdade de gênero, uma vez que o objetivo é fazer uma análise causal consistente, o IED será utilizado com uma defasagem de dois anos no modelo. Também foi utilizado o volume de comércio, que consiste na soma de exportações e importações como proporção do PIB (EX+IM/ PIB). Os dados de comércio exterior foram coletados na plataforma 
Alice Web do Ministério do Desenvolvimento, Indústria e Comércio Exterior (MDIC). Por se encontrarem em dólar, os valores de exportação e importação foram convertidos em real à taxa de câmbio vigente no final do período, obtida no site do Banco Central do Brasil, para depois serem divididos pelo valor do PIB de cada estado.

Na modelagem teórica, como medidas de bem-estar, duas variáveis aparecem como potenciais razões para maior/menor equidade entre os gêneros: a desigualdade socioeconômica (Schneider, 2013) e a pobreza (Bárcena-Martín e Moro-Egido, 2013). Schneider (2013) mostra que a participação diferente no mercado de trabalho para os distintos gêneros ocasiona um aumento na desigualdade entre eles. Quando os homens encontram vagas em empregos ainda mais estáveis e bem remunerados e as mulheres ficam à mercê de empregos de pior qualidade, a desigualdade entre eles aumenta. Segundo Schneider (2013), “(...) White women have experienced the most complicated evolution in their earnings, because (...) the distance between them [women] and top white male income earners grew." (Schneider, 2013, p. 501).

Um indicador correlacionado a essa piora de bem-estar no mercado de trabalho é o indicador de pobreza. Bárcena-Martín e Moro-Egido (2013) apontam que o crescimento da pobreza conduz a um aumento da desigualdade até mesmo por famílias chefiadas por mulheres não casadas serem mais representativas na parcela de menor poder aquisitivo da população. Usando hipóteses de capital humano, distribuição etária, segregação ocupacional e estrutura familiar como fatores microeconômicos de indicação de pobreza, Bárcena-Martín e Moro-Egido (2013) reconhecem que as características pessoais - via os fatores mencionados - são extremamente relevantes na explicação do crescimento da pobreza e, mais do que isso, do gap de pobreza entre os gêneros que segue uma trajetória de crescimento.

No modelo empírico, as medidas de bem-estar escolhidas foram: a taxa de extrema pobreza, o coeficiente de Gini e os gastos correntes do governo. ${ }^{7}$ Todas essas variáveis foram coletadas por unidade da

\footnotetext{
7 Os revisores deste artigo apontaram a necessidade de testarmos outras variáveis que tivessem impacto na questão de bem-estar social. Baseados nessa sugestão, testamos uma variável que indicasse a diferença na precariedade de inserção de homens e mulheres no mercado de trabalho. A variável consistia na razão entre o percentual de mulheres e homens inseridos em trabalhos sem carteira assinada com base em dados da PNAD. Entretanto, ao testarmos o impacto dessa variável nas diferentes regressões, em nenhuma delas obtivemos significância estatística nem melhora dos modelos estimados que justificasse sua permanência nos modelos.
} 
federação no Ipeadata para os anos 1997, 2002, 2007 e 2012. A linha de extrema pobreza considerada para o cálculo da taxa pelo IBGE é uma estimativa do valor monetário de uma cesta de alimentos com o mínimo de calorias necessárias para suprir adequadamente uma pessoa, com base em recomendações da Organização da Comida e Agricultura (FAO na sigla em inglês) e da Organização Mundial da Saúde (OMS) e, portanto, varia entre os estados. O coeficiente de Gini consiste numa medida do grau de concentração de distribuição de renda, cujo valor varia de zero (perfeita igualdade) até 1 (desigualdade máxima). Os gastos correntes do governo são as despesas correntes do governo do estado deflacionados pelo IPCA.

Por fim, a última variável pertencente ao segmento teórico que implicaria alterações no IIG é o desenvolvimento econômico. Segundo Lagerlöf (2003), os padrões históricos que constituem a sociedade podem permitir uma maior ou menor influência das mulheres nas atividades econômicas, políticas e sociais. Esses fatores seriam modelados através tanto das preferências individuais e dos custos de atividades não produtivas (cuidado com crianças, por exemplo), como pela influência do crescimento econômico via aumento da produtividade de cada um dos gêneros. Nesse modelo, a diferença de capital humano entre homens e mulheres é uma consequência da coordenação dos agentes e pode, segundo algumas hipóteses, modificar o status quo da diferença de gênero. A ambiguidade do efeito dessa variável provém da dificuldade em auferir quais os setores econômicos que estão sendo os motores do crescimento, de maneira similar ao que já foi especificado para fluxo de capitais estrangeiros. Dessa forma, para o modelo empírico, consideramos como proxy para o desenvolvimento econômico o crescimento do Produto Interno Bruto (PIB) de cada unidade da federação. Os dados do PIB de cada estado foram coletados na base de dados do Instituto de Pesquisa Econômica Aplicada (Ipeadata) para os anos de 1995, 2000, 2005 e 2010, que é o último ano disponível. Eles foram, então, deflacionados pelo Índice de Preços ao Consumidor Amplo (IPCA). Por fim, calculou-se a taxa de crescimento do PIB em cinco anos, de modo que essa variável entrou com uma defasagem de dois anos no modelo pelos mesmos motivos que o IED também foi defasado.

A Tabela 2 contém a descrição das variáveis explicativas utilizadas neste trabalho, o sinal esperado dos coeficientes e os principais artigos científicos em que essas variáveis foram usadas. Para analisar 
mais especificamente o índice de igualdade de gênero, foram estimadas outras quatro regressões com dados em painel, considerando como variável resposta cada uma das quatro categorias em que se divide o IIG - Participação Econômica, Educação, Poder Político e Saúde/Sobrevivência- do estado $i$ no ano $t$. Com isso, buscamos investigar como as variáveis explicativas impactam cada uma dessas quatro categorias do IIG independentemente e como elas se inter -relacionam resultando no impacto final sobre o índice.

Tabela 2 - Descrição das variáveis explicativas e seus efeitos esperados.

\begin{tabular}{|c|c|c|c|}
\hline Variável & Proxy & $\begin{array}{l}\text { Efeito } \\
\text { esperado }\end{array}$ & Fonte \\
\hline & $\begin{array}{c}\text { Investimento } \\
\text { estrangeiro direto }\end{array}$ & \multirow[t]{4}{*}{ Incerto } & Oostendorp (2004); \\
\hline & $E X+I M / P I B$ & & Juhn, Ujhelyi, e Villegas- \\
\hline & $\begin{array}{c}\text { Percentual de domicílios } \\
\text { com televisores }\end{array}$ & & Sanchez (2013); La Ferrara, \\
\hline & $\begin{array}{l}\text { Percentual de divórcios soli- } \\
\text { citados pela mulher em casos } \\
\text { de divórcio não consensual }\end{array}$ & & $\begin{array}{c}\text { Arora (2012); Mukhopadhyay } \\
\text { e Chaudhuri (2013) }\end{array}$ \\
\hline \multirow{3}{*}{ Bem-estar } & $\begin{array}{l}\text { Percentual de pessoas } \\
\text { vivendo abaixo da linha } \\
\text { da extrema pobreza }\end{array}$ & \multirow{3}{*}{+} & Schneider (2013); \\
\hline & Índice de Gini & & Moro-Egido(2013); \\
\hline & Gastos do Governo & & Seguino (2006) \\
\hline $\begin{array}{l}\text { Desenvolvimento } \\
\text { Econômico }\end{array}$ & Crescimento do PIB & Incerto & $\begin{array}{c}\text { Dollar and Gatti } \\
\text { (1999); Gumbel (2004); } \\
\text { Lagerlöf(2003) }\end{array}$ \\
\hline
\end{tabular}

Fonte: elaboração própria.

As variáveis dummy foram incluídas no modelo para cada ano da amostra a fim de controlar pelo efeito específico de cada ano comum a todas as unidades da federação - o ano 1997 é o ano de referência. Essas variáveis são importantes, uma vez que controlam pelo efeito de variações no IIG devido a algum choque que afete a economia brasileira como um todo.

Ao final da modelagem foi realizada uma análise de resíduos cuidadosa para verificar a validade das suposições do modelo de regressão, em especial a homocedasticidade e normalidade dos erros. ${ }^{8}$

\footnotetext{
8 Mais detalhes em Wooldridge (2003).
} 


\section{Resultados}

\subsection{Análise descritiva}

A distribuição do IIG entre os estados brasileiros no ano de 2012 pode ser observada na Figura 1. Os estados foram separados em quartis amostrais, dividindo as unidades da federação brasileiras em quatro grupos: menor do que 0,695 (estados com menor igualdade de gênero), entre 0,695 e 0,708, entre 0,708 e 0,726 e maior que 0,762 (estados com maior igualdade de gênero). Existe uma razoável discrepância entre os dados de igualdade de gêneros dos estados brasileiros.

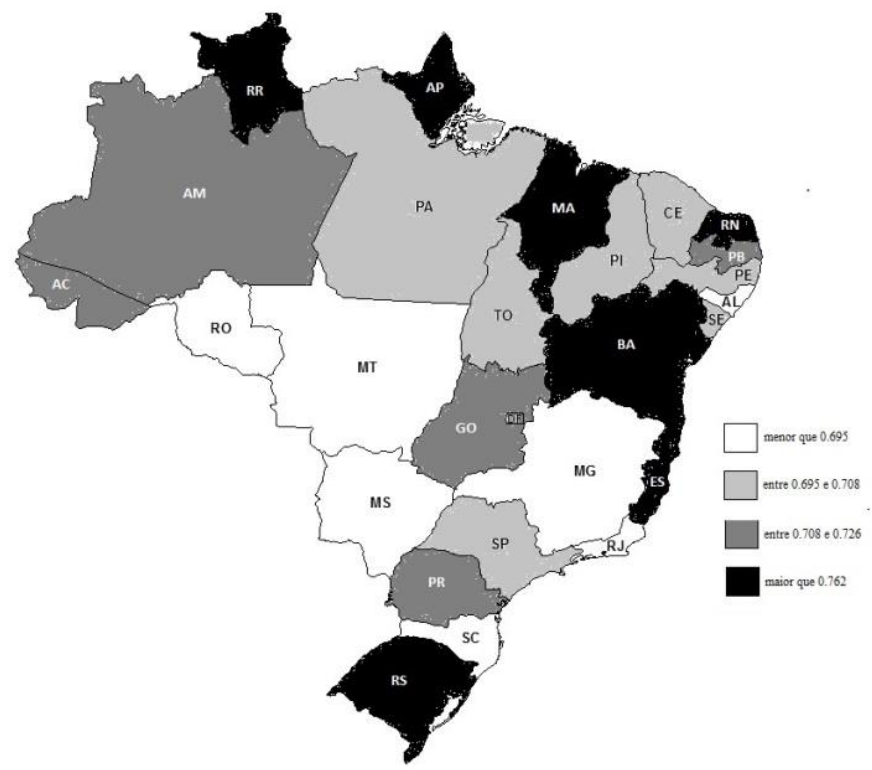

Figura 1 - Distribuição do IIG entre os estados brasileiros em 2012.

Fonte: elaboração própria.

Os estados do Maranhão $(0,81)$, Amapá $(0,80)$, Bahia $(0,78)$ e Rio Grande do Norte $(0,77)$ apresentaram maior igualdade entre homens e mulheres em 2012, enquanto os estados de Mato Grosso $(0,67)$, Minas Gerais $(0,68)$, Alagoas $(0,69)$ e Rondônia $(0,69)$ possuíam a menor igualdade entre os gêneros. Observando-se a Figura 1, percebe-se que, mesmo estados considerados mais desenvolvidos, como Rio de Janeiro, São Paulo e Santa Catarina, figuram entre aqueles 
com maior desigualdade entre os gêneros. Vale a pena destacar nessa figura que os estados com maiores índices no IIG são exatamente aqueles que têm um melhor indicador no subitem de igualdade entre os gêneros na política, isto é, onde a participação de mulheres em cargos escolhidos via eleições é mais evidente.

Para descrever as características da amostra, a Tabela 3 contém uma análise descritiva dos dados. Na média, observa-se um pequeno aumento do IIG nos últimos 15 anos, que passou de 0,68 em 1997 para 0,72 em 2012. Os estados do Norte e Nordeste, no geral, possuem um melhor desempenho no índice, de modo que o valor máximo do IIG na amostra, 0,85, corresponde ao estado do Rio Grande do Norte em 2007. Por outro lado, os estados do Sul e Sudeste apresentam piores resultados para o IIG, com o estado de Santa Catarina apresentando o menor valor da amostra, 0,64, em 1997 e 2002. Portanto, analogamente ao concluído por Ramos e Souza (2012), renda não parece ser um fator determinante para uma maior equidade de gênero.

As variáveis utilizadas como proxy para globalização mostraram um avanço considerável no período analisado, com exceção do percentual de divórcios não consensuais requerido pela mulher, que avançou 3 pontos percentuais, na média, entre 1997 e 2012. Porém, todas elas variam significativamente entre as unidades da federação e apresentam, no geral, melhores resultados nas regiões mais desenvolvidas do país. O Paraná, entretanto, possui baixíssimo coeficiente de abertura comercial, e o Amazonas apresenta alto valor para o mesmo por causa dos incentivos na Zona Franca de Manaus. São Paulo possui o valor máximo da amostra para as variáveis IED, razão de exportações mais importações pelo PIB e proporção de domicílios com acesso a televisores em todos os anos em análise, enquanto estados como Tocantins, Acre e Roraima figuram entre os menores valores. Já o percentual de divórcios não consensuais solicitado pela mulher apresenta valores elevados em estados da região Centro-Oeste, como Mato Grosso do Sul e Goiás, e também no estado de São Paulo, mas valores mínimos em estados como Acre e Maranhão. 
Tabela 3 - Análise descritiva das variáveis por ano avaliado.

\begin{tabular}{|c|c|c|c|c|c|c|}
\hline Variável & Ano & Média & $\begin{array}{l}\text { Desvio } \\
\text { padrão }\end{array}$ & Mínimo & Máximo & Observações \\
\hline \multirow{5}{*}{$\begin{array}{l}\text { Índice Nacional de I } \\
\text { gualdade de Gênero }\end{array}$} & 1997 & 0,68 & 0,04 & 0,64 & 0,78 & 27 \\
\hline & 2002 & 0,68 & 0,03 & 0,64 & 0,77 & 27 \\
\hline & 2007 & 0,71 & 0,04 & 0,64 & 0,85 & 27 \\
\hline & 2012 & 0,72 & 0,04 & 0,67 & 0,81 & 27 \\
\hline & Total & 0,70 & 0,04 & 0,64 & 0,85 & 81 \\
\hline \multirow{5}{*}{$\begin{array}{c}\text { IED } \\
\text { (mil US\$) }\end{array}$} & 1995 & $1.127,64$ & $3.917,00$ & 0 & $20.251,25$ & 27 \\
\hline & 2000 & $3.145,85$ & $10.915,42$ & 0,51 & $56.132,72$ & 27 \\
\hline & 2005 & $5.663,24$ & $17.371,79$ & 4,07 & $82.285,35$ & 27 \\
\hline & 2010 & $3.811,57$ & $13.225,31$ & 0.62 & $68.011,36$ & 27 \\
\hline & Total & 189,91 & 358,99 & 0 & $2.775,37$ & 81 \\
\hline \multirow{5}{*}{$\begin{array}{c}\text { (Exportações + } \\
\text { Importações)/PIB } \\
(\%)\end{array}$} & 1997 & 9,84 & 10,67 & 0,23 & 42,99 & 27 \\
\hline & 2002 & 18,42 & 17,51 & 0,24 & 67,23 & 27 \\
\hline & 2007 & 13,68 & 11,32 & 0,11 & 39,64 & 27 \\
\hline & 2012 & 20,90 & 17,33 & 0,34 & 52,94 & 27 \\
\hline & Total & 15,71 & 14,33 & 0,11 & 67,23 & 81 \\
\hline \multirow{6}{*}{$\begin{array}{l}\text { Domicílios com TV coloridas } \\
\qquad(\%)\end{array}$} & 1997 & 67,5 & 14,32 & 38,65 & 92,23 & 27 \\
\hline & 2002 & 81,09 & 9,99 & 60,45 & 96,29 & 27 \\
\hline & 2007 & 90,64 & 5,27 & 78,75 & 98,64 & 27 \\
\hline & 2012 & 95,83 & 2,22 & 91,16 & 98,97 & 27 \\
\hline & Total & 83,77 & 8,02 & 38,65 & 98,97 & 81 \\
\hline & 1997 & 51,70 & 9,17 & 28,39 & 65,67 & 27 \\
\hline \multirow{4}{*}{$\begin{array}{c}\text { Divórcios Não Consensuais } \\
\text { Solicitado por Mulher } \\
(\%)\end{array}$} & 2002 & 52,70 & 6,69 & 38,00 & 61,41 & 27 \\
\hline & 2007 & 51,15 & 5,41 & 41,53 & 63,09 & 27 \\
\hline & 2012 & 54,90 & 4,78 & 46,30 & 62,41 & 27 \\
\hline & Total & 52,33 & 6,92 & 28,39 & 65,67 & 81 \\
\hline \multirow{5}{*}{$\begin{array}{l}\text { Pessoas vivendo abaixo da } \\
\text { linha da extrema pobreza } \\
(\%)\end{array}$} & 1997 & 19,16 & 12,41 & 4,87 & 44,98 & 27 \\
\hline & 2002 & 17,39 & 9,99 & 3,44 & 36,03 & 27 \\
\hline & 2007 & 11,61 & 7,40 & 1,55 & 23,85 & 27 \\
\hline & 2012 & 6,28 & 4,18 & 1,40 & 17,71 & 27 \\
\hline & Total & 13,61 & 10,25 & 1,40 & 44,98 & 81 \\
\hline \multirow{5}{*}{ Índice de Gini } & 1997 & 0,58 & 0,04 & 0,44 & 0,63 & 27 \\
\hline & 2002 & 0,57 & 0,03 & 0,47 & 0,63 & 27 \\
\hline & 2007 & 0,54 & 0,04 & 0,46 & 0,61 & 27 \\
\hline & 2012 & 0,52 & 0,04 & 0,42 & 0,61 & 27 \\
\hline & Total & 0,55 & 0,04 & 0,42 & 0,63 & 81 \\
\hline \multirow{5}{*}{$\begin{array}{l}\text { Gastos do governo } \\
\text { (mil R\$) }\end{array}$} & 1997 & 594,99 & 956,78 & 43,97 & $4.888,10$ & 27 \\
\hline & 2002 & $2.446,68$ & $3.986,24$ & 217,55 & $10.130,60$ & 27 \\
\hline & 2007 & $6.959,77$ & $10.526,53$ & 653,38 & $52.117,14$ & 27 \\
\hline & 2012 & $16.167,47$ & $24.498,41$ & $1.748,17$ & $126.050,05$ & 27 \\
\hline & Total & $6.542,23$ & $14.609,20$ & 43,97 & $126.050,05$ & 81 \\
\hline \multirow{5}{*}{$\begin{array}{l}\text { Crescimento do PIB } \\
\text { (\% em } 5 \text { anos) }\end{array}$} & 1995 & 18,59 & 15,66 & $-33,8$ & 41,38 & 27 \\
\hline & 2000 & 21,32 & 11,53 & $-1,35$ & 46,73 & 27 \\
\hline & 2005 & 42,26 & 27,41 & 10,65 & 134,19 & 27 \\
\hline & 2010 & 41,48 & 7,52 & 20,14 & 56,20 & 27 \\
\hline & Total & 30,91 & 20,26 & $-33,8$ & 134,19 & 81 \\
\hline
\end{tabular}

Fonte: elaboração própria. 
As medidas de bem-estar também evoluíram consideravelmente entre 1997 e 2012. O índice de Gini passou, na média, de 0,58 para 0,52 , apontando para uma queda da desigualdade de renda no país neste período. Os estados menos desiguais concentram-se nas regiões Sul-Sudeste do Brasil, como Santa Catarina, São Paulo e Rio Grande do Sul, enquanto aqueles com uma maior disparidade de renda localizam-se no Norte-Nordeste, como Alagoas, Sergipe e Maranhão. Os gastos do governo apresentaram a maior variação no período, passando, na média, de R \$594,9 mil em 1997 para R \$16.167,47 em 2012. Novamente, os estados com os menores e maiores gastos são aqueles das regiões Norte-Nordeste e Sul-Sudeste respectivamente.

Com relação ao desenvolvimento econômico dos estados brasileiros, mensurado pelo crescimento percentual do PIB em 5 anos, observase, na média, um aumento significativo com avanço de 18,59\% em 1995 para 41,48\% em 2010. A distribuição deste crescimento entre as regiões brasileiras acontece de modo bastante heterogêneo, com anos em que estados como Tocantins, Roraima e Mato Grosso foram os que mais cresceram, enquanto em outros anos os mesmos foram os que menos cresceram.

\subsection{Análise econométrica}

Para estimar o efeito das variáveis que medem globalização, bem-estar e desenvolvimento econômico na igualdade de gêneros foi utilizado o modelo de regressão com dados em painel. O teste de Hausman indicou os estimadores de efeitos aleatórios são consistentes e mais eficientes que os de efeito fixo e, portanto, foram escolhidos para a análise dos resultados. Em todos os modelos foi empregada a correção de heterocedasticidade de White em cross section. A Tabela 4 contém os resultados de todas as regressões por efeitos aleatórios. $\mathrm{Na}$ coluna IIG total temos o resultado para o índice todo e nas demais colunas separamos o índice por dimensão: economia, educação, política e saúde.

Dentre as variáveis utilizadas como proxy para globalização, o investimento estrangeiro direto e o percentual de domicílios com acesso à televisão mostraram-se estatisticamente relevantes para explicar a distribuição da igualdade de gênero entre os estados brasileiros, além de indicar que aumentos nessas variáveis diminuem a desi- 
gualdade de gênero. No geral, os resultados mostram que estados mais globalizados são mais avançados quanto à igualdade entre os sexos, corroborando com a literatura apresentada. Os resultados de La Ferrara, Chong e Duryea (2012) e Arora (2012) apontavam para casos específicos onde o maior contato com a cultura globalizada implicava em mudanças de comportamento sociais ou de escolhas individuais. ${ }^{9}$ Uma das explicações para esse resultado está na coluna seguinte, onde se encontram os resultados relativos à dimensão "Economia" do IIG. A significância do coeficiente ligado ao percentual de domicílios com acesso à televisão só ocorre para essa dimensão do índice, indicando que, com maiores níveis de informações globalizadas, existe uma maior conscientização da população sobre a importância da igualdade, além de fomentar a economia, gerando mais empregos, por exemplo. Também é interessante ressaltar que as variáveis relativas ao Investimento Estrangeiro Direto (IED) e de grau de abertura comercial (EX+IM/PIB) são muito significantes para a dimensão "Política" do IIG. Os impactos de IED e de abertura comercial no subíndice de política corroboram com os resultados encontrados por Dollar e Gatti (1999) e Gumbel (2004) no sentido em que medidas que impactam positivamente a renda per capita têm potencial impacto positivo na igualdade de gênero e, em nossa amostra, esse impacto é mais relevante na dimensão de participação política das mulheres do que nas demais dimensões. Essas duas variáveis implicam um aumento significativo da igualdade política entre homens e mulheres. Com maior participação feminina na esfera pública, a desigualdade na tomada de decisões diminui e, consequentemente, melhora a distribuição de poder entre os gêneros. No mesmo sentido, investimentos estrangeiros que ocasionam a melhoria na produtividade de mão de obra feminina também têm impacto na diminuição das diferenças entre os gêneros, seguindo os resultados de Mukhopadhyay e Chaudhuri (2013) e Juhn, Ujhelyi e Villegas-Sanches (2013), este último para dados comparativos do México com os Estados Unidos.

Embora o percentual de pedidos de divórcio não consensual feito por mulheres tenha sido incluso na regressão, essa variável não obteve impacto significativo no IIG. Mas, analisando separadamente por dimensões, percebe-se que essa variável tem um impacto positivo

9 La Ferrara, Chong e Duryea (2012) implicava em escolhas relativas à fertilidade, um fator considerado de empoderamento feminino quando a mulher tem a sua disposição métodos contraceptivos e pode optar pelo uso deles sem sanções sociais devido a sua escolha. 
na dimensão educação. Ou seja, quanto mais a cultura vigente no estado permite que as mulheres tomem a iniciativa de sair de um casamento insatisfatório, maior é o impacto na qualificação do capital humano das mulheres e menor a desigualdade entre os gêneros nessa dimensão.

Tabela 4 - Resultados das regressóes com dados em painel por efeitos aleatórios.

\begin{tabular}{|c|c|c|c|c|c|c|}
\hline \multicolumn{7}{|c|}{ Variável Dependente } \\
\hline Dimensão & $\begin{array}{c}\text { Variável } \\
\text { explicativa }\end{array}$ & IIG Total & $\begin{array}{c}\| I G \\
\text { Economia }\end{array}$ & $\begin{array}{c}\text { IIG } \\
\text { Educação }\end{array}$ & IIG Politico & IIG Saúde \\
\hline \multirow{4}{*}{ Globalização } & $\operatorname{Ln}(\mathrm{IED})$ & $\begin{array}{l}0,002^{\star} \\
(0,001)\end{array}$ & $\begin{array}{c}0,003 \\
(0,005)\end{array}$ & $\begin{array}{c}-0,003^{\star \star \star} \\
(0,001)\end{array}$ & $\begin{array}{l}0,012^{\star *} \\
(0,005)\end{array}$ & $\begin{array}{l}-0,002 \\
(0,002)\end{array}$ \\
\hline & $\begin{array}{c}\mathrm{Ln} \\
(\mathrm{EX}+\mathrm{IM} / \mathrm{PIB})\end{array}$ & $\begin{array}{c}0,001 \\
(0,001)\end{array}$ & $\begin{array}{l}-0,005^{\star} \\
(0,002)\end{array}$ & $\begin{array}{l}-0,004^{*} \\
(0,002)\end{array}$ & $\begin{array}{c}0,013^{\star \star \star} \\
(0,004)\end{array}$ & $\begin{array}{l}-0,000 \\
(0,000)\end{array}$ \\
\hline & $\begin{array}{c}\text { TV } \\
\text { \% Divórcios }\end{array}$ & $\begin{array}{c}0,069^{\star * *} \\
(0,021)\end{array}$ & $\begin{array}{l}0,124^{\star \star \star} \\
(0,031)\end{array}$ & $\begin{array}{c}0,045 \\
(0,051)\end{array}$ & $\begin{array}{c}0,112 \\
(0,110)\end{array}$ & $\begin{array}{l}-0,004 \\
(0,025)\end{array}$ \\
\hline & $\begin{array}{c}\text { Não } \\
\text { Consensuais } \\
\text { solicitados por } \\
\text { mulher }\end{array}$ & $\begin{array}{l}-0,053 \\
(0,046)\end{array}$ & $\begin{array}{l}-0,054 \\
(0,067)\end{array}$ & $\begin{array}{l}0,054^{\star \star \star} \\
(0,015)\end{array}$ & $\begin{array}{l}-0,068 \\
(0,233)\end{array}$ & $\begin{array}{l}-0,036 \\
(0,037)\end{array}$ \\
\hline \multirow{3}{*}{ Bem-estar } & Pobreza & $\begin{array}{l}0,082^{*} \\
(0,049)\end{array}$ & $\begin{array}{c}0,283^{\star \star \star} \\
(0,052)\end{array}$ & $\begin{array}{c}0,012 \\
(0,056)\end{array}$ & $\begin{array}{c}0,060 \\
(0,143)\end{array}$ & $\begin{array}{c}0,028 \\
(0,038)\end{array}$ \\
\hline & Gini & $\begin{array}{c}0,121 \\
(0,109)\end{array}$ & $\begin{array}{c}0,198 \\
(0,283)\end{array}$ & $\begin{array}{l}-0,171 \\
(0,129)\end{array}$ & $\begin{array}{c}0,407 \\
(0,520)\end{array}$ & $\begin{array}{l}0,053^{\star *} \\
(0,026)\end{array}$ \\
\hline & $\begin{array}{l}\text { Ln (Gastos do } \\
\text { governo) }\end{array}$ & $\begin{array}{c}-0,007^{\star *} \\
(0,003)\end{array}$ & $\begin{array}{l}-0,007 \\
(0,007)\end{array}$ & $\begin{array}{l}0,005^{*} \\
(0,003)\end{array}$ & $\begin{array}{c}-0,032^{\star \star *} \\
(0,010)\end{array}$ & $\begin{array}{c}0,006 \\
(0,004)\end{array}$ \\
\hline $\begin{array}{l}\text { Desenvolvimento } \\
\text { Econômico }\end{array}$ & Crescimento & $\begin{array}{l}-0,005 \\
(0,013)\end{array}$ & $\begin{array}{l}-0,019 \\
(0,026)\end{array}$ & $\begin{array}{l}0,012^{\star \star *} \\
(0,004)\end{array}$ & $\begin{array}{l}-0,036 \\
(0,051)\end{array}$ & $\begin{array}{c}0,019 \\
(0,015)\end{array}$ \\
\hline \multirow{6}{*}{ Dummies de tempo } & Dummy 2002 & $\begin{array}{l}0,011^{\star} \\
(0,006)\end{array}$ & $\begin{array}{l}0,099^{\star \star \star} \\
(0,004)\end{array}$ & $\begin{array}{c}-0,051^{\star \star \star} \\
(0,009)\end{array}$ & $\begin{array}{c}0,007 \\
(0,029)\end{array}$ & $\begin{array}{c}-0,011^{\star \star \star} \\
(0,004)\end{array}$ \\
\hline & Dummy 2007 & $\begin{array}{c}0,046^{\star \star *} \\
(0,016)\end{array}$ & $\begin{array}{c}0,131^{\star \star *} \\
(0,015)\end{array}$ & $\begin{array}{l}-0,024 \\
(0,017)\end{array}$ & $\begin{array}{c}0,103 \\
(0,069)\end{array}$ & $\begin{array}{c}-0,025^{\star \star \star} \\
(0,009)\end{array}$ \\
\hline & Dummy 2012 & $\begin{array}{c}0,066^{\star \star \star} \\
(0,020)\end{array}$ & $\begin{array}{l}0,159^{\star \star \star} \\
(0,025)\end{array}$ & $\begin{array}{l}-0,039^{*} \\
(0,022)\end{array}$ & $\begin{array}{l}0,171^{\star *} \\
(0,086)\end{array}$ & $\begin{array}{l}-0,025^{\star} \\
(0,014)\end{array}$ \\
\hline & Constante & $\begin{array}{c}0,645^{\star \star \star} \\
(0,083)\end{array}$ & $\begin{array}{l}0,513^{\star \star} \\
(0,251)\end{array}$ & $\begin{array}{l}1,046^{\star * \star} \\
(0,040)\end{array}$ & $\begin{array}{c}0,125 \\
(0,336)\end{array}$ & $\begin{array}{c}0,896^{\star * *} \\
(0,049)\end{array}$ \\
\hline & $R^{2}$ & 0,320 & 0,634 & 0,454 & 0,133 & 0,187 \\
\hline & Observações & 107 & 107 & 107 & 107 & 107 \\
\hline
\end{tabular}

Nota: ${ }^{* * *}$ Significante a $1 \% ;{ }^{* *}$ Significante a $5 \% ;{ }^{*}$ Significante a $10 \%$; erros padrão robustos entre parênteses.

Fonte: Elaboração própria. 
Dentre as variáveis que mensuram o bem-estar de um determinado estado, maiores gastos do governo estadual implicam uma diminuição do IIG, o aumento do percentual de pessoas vivendo sob extrema pobreza aumentam a igualdade entre homens e mulheres e, por fim, o índice de Gini não se mostra estaticamente relevante. Os resultados vão de encontro àqueles encontrados na literatura (Seguino, 2006). Também nessa dimensão é interessante observar os resultados parciais das variáveis em cada uma das dimensões do IIG. Com relação ao indicador de pobreza (percentual de pessoas vivendo sob extrema pobreza), ele somente se mostra significante e positivo quando usado para explicar o IIG da dimensão econômica, o que significa diminuir a diferença entre homens e mulheres na esfera econômica. Uma das potenciais explicações para esse resultado está baseada em estatísticas referentes a diferenças salariais entre os gêneros. Diversos estudos apontam (Frolich, 2007; Madalozzo e Martins, 2007) que a maior diferença de remuneração entre homens e mulheres ocorre para indivíduos com alto nível de educação e com nível hierárquico elevado dentro das empresas. Utilizando dados do Ministério do Trabalho e Emprego (2012), percebe-se que a diferença salarial entre homens e mulheres analfabetos é, em média, 17\%; ao mesmo tempo, a diferença salarial média entre homens e mulheres com ensino superior é de $40 \% .{ }^{10} \mathrm{Ou}$ seja, a extrema pobreza torna mais iguais os trabalhadores de diferentes gêneros e, com isso, reduz significativamente o índice calculado.

O desenvolvimento econômico, medido pelo crescimento percentual do PIB em 5 anos, não se mostrou estatisticamente significante. Entretanto, analisando novamente o impacto por diferentes dimensões, o crescimento econômico leva a uma melhoria no índice de IIG para a dimensão educação. Esse resultado está alinhado àquele a que Dollar e Gatti (1999) e Gumbel(2004) chegaram: o crescimento e seu consequente aumento de renda per capita fazem com que as diferenças de formação de capital humano entre os gêneros diminuam. Como o reflexo de melhorias no capital humano (mais especificamente, educação) consomem mais tempo para refletirem em outras áreas (como remuneração, por exemplo), é possível que, dentro do período analisado, o impacto esteja se revelando somente em uma primeira ordem, dentro da dimensão educação, para ter reflexo em médio ou longo prazo nas outras dimensões e no próprio índice agregado.

${ }^{10}$ Fonte: Rais, 2012, Ministério do Trabalho e Emprego. 
As dummies de tempo, utilizadas para controlar por efeitos que afetam a igualdade de gênero, mas que não são capturados pelas variáveis explicativas, indicam que a igualdade de gênero no Brasil aumentou no período analisado. Nota-se que os coeficientes aumentam de tamanho ao longo do tempo e que todos são estatisticamente significantes, indicando o aumento da igualdade entre homens e mulheres no período avaliado e de acordo com os resultados do Fórum Econômico Mundial para esse período.

Embora os resultados anteriormente citados estejam bastante alinhados com a literatura que os precede, também fizemos testes de robustez com relação a dois índices: o INDG (proposto por Ramos e Souza, 2012) e o Gender Inequality Index (do PNUD, 2010, e modificado por Permanyer, 2013). Os resultados foram similares para os três índices utilizados tanto em termos de sinal como significância estatística. Para o INDG, houve a significância da taxa de divórcios, significando que o aumento do número de pedidos de divórcios por parte das mulheres aumenta a igualdade de gêneros, enquanto o percentual de TV colorida perdeu a relevância estatística. No caso do Gender Inequality Index, o índice de Gini foi relevante, isto é, quanto maior a desigualdade de renda, maior a desigualdade de gêneros e, em contrapartida, o investimento direto estrangeiro perdeu a significância. Os resultados estão disponíveis mediante solicitação aos autores.

\section{Conclusão}

A igualdade entre homens e mulheres é uma das condições para que o crescimento sustentável se torne realidade. O Brasil, embora seja uma das maiores economias do mundo (FMI, 2013), ainda está em uma colocação bastante distante da igualdade entre os gêneros quando comparado com diversas outras economias no mundo $\left(71^{\circ}\right.$. lugar de acordo com o ranqueamento de 2014 do Fórum Econômico Mundial). O presente estudo aplicou a metodologia de dados em painel para analisar os determinantes da distribuição da desigualdade de gênero, mensurada pelo Índice de Igualde de Gêneros (IIG), entre os diferentes estados brasileiros no período de 1997 a 2012. 
Os resultados das estimações mostram que a globalização, medida pelo investimento estrangeiro direto, pela soma de exportações e importações pelo PIB e pelo percentual de domicílios com televisores reduzem a desigualdade de gênero. Por outro lado, as medidas de bem-estar (percentual de pessoas vivendo abaixo da linha da extrema pobreza e gastos do governo estadual) elevam a desigualdade entre os sexos. As outras variáveis que implicam abertura cultural ou maior desenvolvimento econômico não são estatisticamente relevantes para explicar a distribuição da desigualdade de gênero entre os estados brasileiros.

Uma importante contribuição deste trabalho foi analisar a evolução da desigualdade de gênero no Brasil entre 1997 e 2012 por meio da construção de um índice que engloba diferentes dimensões em que persiste a realidade da desigualdade entre os gêneros. Além disso, foram analisados os determinantes dos diferentes níveis de desigualdade de gênero existente entre os estados brasileiros, algo inédito na literatura.

Apesar da queda da desigualdade observada tanto no presente trabalho como no ranking do Fórum Econômico Global, a diferença de tratamento de acordo com os gêneros no Brasil continua em níveis elevados. Portanto, é importante incentivar a formulação de politicas públicas que promovam a igualdade nos direitos de liberdade e de oportunidades entre elas e os homens, levando o Brasil ao desenvolvimento sustentável e a níveis de igualdade de gênero compatíveis com um país de seu porte econômico.

\section{Referências}

ADDISON, J. T., \& DEMET OZTURK, O. Minimum Wages, Labor market Institutions, and Female Employment: a cross-country analysis. Industrial \& Labor Relations Review, v. 65, n.4, p. 779809, 2012.

ARORA, R. Gender Inequality, Economic Development, and Globalization: a State Level Analysis of India. The Journal of Developing Areas, v. 46, n. 1, p. 148-164, 2012.

BANCO MUNDIAL, 2014. Disponível em http://www.worldbank.org/mdgs/gender.html.

BALIAMOUNE-LUTZ, M. Globalisation and Gender: Is Africa Different? Journal of African Economies, v. 16, n. 2, p. 301-348, 2006.

BÁRCENA-MARTÍN, E., MORO-EGIDO, A. Gender and Poverty Risk in Europe. Feminist Economics, v. 19, n. 2. p. 69-99, 2013. 
BORTOLUZZO, M., SAKURAI, S., BORTOLUZZO, ADRIANA B. Allocation of Foreign Direct Investment across Brazilian States. Estudos Econômicos, v. 43, n. 2, p. 241-269, 2013.

BRANISA, B., KLASEN, S., ZIEGLER, M., DRESCHSLER, D., \& JUTTING, J. The Institutional Basis of Gender Inequality: The Social Institutions and Gender Index (SIGI). Feminist Economics, v. 20, n. 2, p. 29-64, 2014.

DOLLAR, D.; \& GATTI, R. Gender Inequality, Income, and Growth: Are Good Times Good for Women? Policy Research Report on Gender and Development: Working Paper Series, n.1, Washington DC: World Bank, 1999.

FMI. World Economic Outlook Database. Fundo Monetário Internacional, 2013.

FOLBRE, N. Measuring Care: Gender, Empowerment, and the Care Economy. Journal of Human Development, v.10, n.2, p. 183-199, 2006.

FÓRUM ECONÔMICO MUNDIAL. Global Gender Gap Report, 2013.

FROLICH, M. Propensity Score Matching without Conditional Independence Assumption--With an Application to the Gender Wage Gap in the United Kingdom. Econometrics Journal, v. 10, n. 2, p. 359-407, 2007

GIUBERTI, A.; MENEZES-FILHO, N. Discriminação dos rendimentos por gênero: uma comparação entre o Brasil e os Estados Unidos. Economia Aplicada, v.9, n.3, p.369-383, 2005.

GUMBEL, D. The Influence of Gender Inequality on Economic Growth, 2004. Disponível em http:// david-guembel.de/uploads/media/Ausarbeitung_Seminar_Wirtschaftsgeschichte_Gender_Inequality.pdf

HAM, J. C., \& SONG, H. The Determinants of Bargaining Power in an Empirical Model of Transfers between Adult Children, Parents, and In-Laws for South Korea. Journal of Development Economics, v. 109, p. 10973-86, 2014.

JUHN, C.; UJHELYI, G.; VILLEGAS-SANCHES, C. Trade Liberalization and Gender Inequality. American Economic Review, v.13, n. 3, p. 269-273, 2013.

KNOWLES, S.; LORGELLY, P.; OWEN, P. Are educational gender gaps a brake on economic development? Some cross-country empirical evidence. Oxford Economic Papers, v. 54, p.118-149, 2002.

LA FERRARA, E., CHONG, A. e DURYEA, S. Soap operas and fertility: Evidence from Brazil. American Economic Journal: Applied Economics, v. 4, n. 4, p. 1-31, 2012.

LAGERLÖF, N. Gender Equality and Long-Run Growth. Journal of Economic Growth, v. 8, p. 403426, 2003.

MADALOZZO, R. Occupational segregational and the gender wage gap in Brazil: an empirical analysis. Economia aplicada, v. 14, n. 2, p.147-168, 2010.

MADALOZZO, R. e MARTINS, S. Gender wage gaps: comparing the 80s, the 90s and 00s in Brazil, Revista de Economia e Administração, v. 6, n. 2, p. 141-156, 2007.

MIKI, M., \& YUVAL1, F. Using Education to Reduce the Wage Gap between Men and Women. Journal Of Socio-Economics, v. 40, n. 4, p. 412-416, 2011.

MINISTÉRIO DO TRABALHO E EMPREGO (MTE). http://portal.mte.gov.br/data/files/ FF80808148855DD701489F100CA546F5/consolidado\%20tabelas\%20(2).pdf (acessado em $11 / 12 / 14)$.

MUKHOPADHYAY, U. e CHAUDHURI, S. Economic liberalisation, gender wage inequality and welfare, The Journal of International Trade \& Economic Development, v. 22, n. 8, p. 1214-1239, 2013.

OOSTENDORP, R. H. Globalization and the Gender Wage Gap. World Bank Policy Research Working Paper, n. 3256, Washington DC: World Bank, 2004.

PERMANYER, I. A critical assessment of the UNDP's gender inequality index. Feminist Economics, v. 19, n. 3, p. 1-32, 2013. 
RAMOS, Daniela Peixoto. Pesquisas de usos do tempo: um instrumento para aferir as desigualdades de gênero. Revista Estudos Feministas, v. 17, n. 3, p. 861-870, 2009.

RAMOS, C; SOUZA, L. A Mensuração da Desigualdade de Gênero: um Índice para os Estados Brasileiros, 2012. Disponível em http://bdm.unb.br/bitstream/10483/5731/1/2012_LuisaCardosoGuedesdeSouza.pdf

ROSA, Waldemir. Sexo e cor: categorias de controle social e reprodução das desigualdades socioeconômicas no Brasil. Revista Estudos Feministas, v. 17, n. 3, p. 889-899, 2009.

SCHNEIDER, M. Illustrating the Implications of How Inequality is Measured: Decomposing Earnings Inequality by Race and Gender. Journal of Labor Research, v. 34, p. 476-514, 2013.

SEGUINO, S. The great equalizer? Globalization effects on gender equality in Latin America and the Caribbean. Munich Personal Research Paper in Economics Archive, n. 6509, 2006.

SEN, A. Many Faces of Gender Inequality. Frontline, v. 18, n. 22, p.35-40, 2001.

SENADZA, B. Education Inequality in Ghana: Gender and Spatial Dimensions. Journal of Economic Studies, v. 39, n. 5-6, p. 724-739, 2012.

UNDP. Human Development Reports: Gender Inequality Index, 2011. Disponível em http://hdr.undp. org/en/statistics/gii/

UNFPA, 2014. Disponível em: http://www.unfpa.org/gender/

WANG, M., \& KELAN, E. The Gender Quota and Female Leadership: Effects of the Norwegian Gender Quota on Board Chairs and CEOs. Journal of Business Ethics, v. 117, n. 3, p. 449-466, 2013.

WEICHSELBAUMER e WINTER-EBMER. A Meta-Analysis of the International Gender Wage Gap. Journal of Economic Surveys, v. 19, n. 3, p.479-511, 2005.

WOOLDRIDGE, J. M. Introductory Econometrics: A Modern Approach, 2a. edição, Mason, Ohio, Estados Unidos, 2003. 